\title{
El Comité de las Regiones en el futuro de Europa
}

\author{
Antonio Calonge Velázquez \\ Profesor Titular de Derecho Administrativo \\ Universidad de Valladolid
}

Sumario: I. EL FUTURO DE EUROPA A DEBATE. II. LAS ASPIRACIONES Y LOS LOGROS DESDE LA CREACIÓN. 1. La reforma de Ámsterdam. 2. La reforma de Niza. III. LAS REIVINDICACIONES FRENTE AL FUTURO. IV. LA POSICIÓN SOBRE LA CONVENCIÓN. V. LA CONTRIBUCIÓN A LA CONVENCIÓN SOBRE EL FUTURO DE EUROPA. VI. LOS RESULTADOS: EL TRATADO POR EL QUE SE ESTABLECE UNA CONSTITUCIÓN PARA EUROPA. 1. Idéntica regulación: naturaleza jurídica, composición y competencias. 2. Alguna pequeña incorporación. VII. UNA CONSIDERACIÓN FINAL.

\section{EL FUTURO DE EUROPA A DEBATE}

El Consejo Europeo de Niza -el más largo de los que hasta la fecha han tenido lugar, ya que duró los días 7, 8 y 9 de diciembre de 2000 - aprobó, como es de todos conocido, el Tratado de Niza de 26 de febrero de $2001{ }^{1}$, que contiene, entre otras, la declaración número 23, aneja al Acta Final del Tratado, sobre el futuro de la Unión.

La mencionada declaración que, en otro lugar, hemos calificado - aunque un tanto exageradamente- como lo más importante del Tratado ${ }^{2}$, preveía la convocatoria de una Conferencia Intergubernamental en el 2004 para tratar las siguientes cuestiones:

- la forma de establecer y supervisar una delimitación más precisa sobre las competencias entre la Unión Europea y los Estados miembros que respete el principio de subsidiariedad.

- el estatuto de la Carta de Derechos Fundamentales de la Unión Europea, proclamada en Niza, de conformidad con las Conclusiones del Consejo Europeo de Colonia

- la simplificación de los Tratados con el fin de clarificar y facilitar su comprensión, sin cambiar su significado.

- la función de los Parlamentos nacionales en la arquitectura europea.

\footnotetext{
${ }^{1}$ DOCE $n^{\circ} \mathrm{C} 80$, de 10 de marzo de 2001.

2 Calonge Velázquez, Antonio, «El Tratado de Niza y la reforma de las instituciones», La Ley, $\mathrm{n}^{\circ}$ 5432, de 4 de diciembre de 2001, p. 4.
} 
Desde luego, el sistema seguido en las últimas reformas de los Tratados comunitarios de anunciar las siguientes modificaciones no es, en mi opinión, nada bueno porque en realidad lo que denota es la ausencia de un consenso verdadero y el consiguiente retraso en la resolución de los problemas, graves e importantes, que implica una Unión Europea a 27 miembros. Si el Tratado de Ámsterdam resultó, como dijera el que fuera Comisario español Marcelino Oreja, un sonado fracaso en cuanto a la reforma institucional de la Unión ${ }^{3}$, ya que no preparó a la Unión Europea para la ampliación, el texto que surgió de lo que un buen conocedor de la Unión Europea denominó «la batalla de Niza» ${ }^{4}$ no ha cumplido, en mi opinión, de manera satisfactoria, los objetivos para el que ha sido elaborado, pues, como han mostrado algunos autores ${ }^{5}$, los dos principales cometidos que tenía la Cumbre de Niza: por un lado, eliminar los obstáculos que el Tratado de Ámsterdam imponía a la ampliación y, por otro, adoptar las reformas necesarias para asegurar que las instituciones de la Unión Europea continuaran operando con eficiencia y eficacia después de que el número de países miembros llegase a duplicarse, no se han visto logrados ${ }^{6}$.

Siguiendo las Conclusiones de Niza, el Consejo Europeo de Laeken de 14 y 15 de diciembre de 2001, aprobó una importantísima Declaración sobre «El futuro de la Unión Europea» (más conocida como Declaración de Laeken), que abre el camino para una Unión más simple, más firme en la persecución de sus objetivos fundamentales y más presente en el mundo; pero, sobre todo, constituye una invitación a todos a reflexionar sobre el futuro de la Unión Europea planteando de manera abierta los interrogantes a que debemos enfrentarnos para definir nuestro futuro en común y que la Unión Europea deje de caminar - como alguien con acierto ha expresado- con la cara tapada. Desde cómo podemos aumentar la legitimidad democrática

\footnotetext{
${ }^{3}$ Oreja Aguirre, Marcelino, «La revisión institutionnelle de l'Union Européenne», Recueil del Cours de l'Academie de Droit International de La Haye (Recueil des Cours), nº 267, 1997, p. 345.

${ }^{4}$ Elorza, Javier, «La batalla de Niza», El País, 21 de diciembre de 2000.

${ }^{5}$ De la Dehesa, Guillermo, «Las instituciones de la Unión Europea después del Tratado de Niza y el papel de España en la ampliación», El papel de España en una Unión Europea ampliada, Círculo de Empresarios, Madrid, 2001, p.127. Si bien, no todos los autores son de esta opinión, ya que para GuTIÉRREZ ESPADA, Cesáreo, «Una reforma 'difícil pero productiva': la revisión institucional en el Tratado de Niza», $R D C, \mathrm{n}^{\circ} 9,2001$, p. 73, «el objetivo principal y necesario se ha conseguido» y para ANDRÉs Sáenz De Santamaría, P., «La reforma institucional en el Tratado de Niza», GJ, no 211, 2001, pp. 25 y 26 «mal que bien se ha dado respuesta a todas las cuestiones planteadas, por lo que se puede asumir la afirmación de Davis O'Sullivan, Secretario de la Comisión: «It was a difficult but productive Summit which none-the-less shows that Europe is entering a new phase of development with all the associated growings-pains». FonseCA MoRILlo, Francisco J. «De Berlín a Niza: Panorama y lecciones», Suplemento Boletín Europeo Universidad de La Rioja, marzo, 2001, p. 2, por su parte, considera de manera acertada —en mi opinión - que estamos ante el final de un modelo, iniciado en Maastricht y que se agota ahora después de sucesivas reformas, y que el futuro requiere la determinación de la voluntad política sobre lo que se pretende hacer a continuación.

${ }^{6}$ Delors, Jacques, que fuera Presidente de la Comisión durante dos mandatos a finales de los ochenta y principios de los noventa, ha afirmado de manera bastante rotunda que el Tratado de Niza «es un completo fracaso» que «no podrá aplicarse nunca». Cfr. El País de 16 de junio de 2001.
} 


\section{EL COMITÉ DE LAS REGIONES EN EL FUTURO DE EUROPA}

y la transparencia de la Unión, hasta si debemos reducir los instrumentos legislativos de que disponemos, pasando por cómo mejorar el reparto de competencias entre la Unión y los Estados miembros o cómo garantizar la coherencia y la eficacia exterior de la Unión son las cuestiones planteadas para abordar de manera decidida y abierta el futuro del proceso de construcción europea.

Al mismo tiempo, el Consejo Europeo de Laeken decidió, para garantizar una preparación lo más amplia y transparente posible de la próxima Conferencia intergubernamental, convocar una Convención que reuniera a los principales participantes en el debate sobre el futuro de la Unión, que tendrá el cometido de examinar las cuestiones esenciales que plantea el futuro desarrollo de la Unión, investigar las distintas respuestas posibles y, en definitiva, formular propuestas a los representantes de los Gobiernos de los Estados miembros. También acordó que dicha Convención tendría por Presidente al Sr. Valery Giscard D'Estaing y por Vicepresidentes a los Srs. Giulio Amato y Jean Luc Dehane. Y, finalmente, resolvió que los Estados candidatos participarían, también, en los trabajos de esta Convención.

En fin, con la declaración aneja al Acta Final del Tratado de Niza y la posterior Declaración de Laeken el futuro de Europa está puesto encima de la mesa de los principales actores del proceso de integración europea en la Convención abierta, primero, y de los representantes de los Gobiernos de los Estados miembros, después, en la Conferencia intergubernamental de 2004.

Ante este escenario, el Comité de las Regiones se pronunció de manera favorable, desde el primer momento, tanto por el debate abierto como por el foro creado para llevarlo a cabo y, así, ya en la Resolución de 4 de abril de 2001 sobre los «Resultados de la Conferencia Intergubernamental de 2000 y el debate sobre el futuro de la Unión Europea» ${ }^{7}$, que dedicó mayor espacio a esta cuestión que a su opinión sobre el Tratado recién aprobado, respecto del debate que se abría este órgano manifestó que «Acoge favorablemente la 'Declaración sobre el futuro de la Unión', apoya expresamente la intención de los Jefes de Estado y de Gobierno de iniciar inmediatamente un amplio debate sobre la futura evolución de la Unión Europea» y, asimismo, que «Acoge con satisfacción que el Consejo Europeo... quiera aprobar en Laeken una Declaración sobre la preparación y los contenidos de la Conferencia Intergubernamental de 2004». Y, después, también manifestó su parecer favorable al instrumento creado para llevar a cabo dicho debate.

\footnotetext{
7 DOCE n ${ }^{\circ}$ C 253/25, de 12 de septiembre de 2001 (CDR 430/2000 fin).
} 


\section{LAS ASPIRACIONES Y LOS LOGROS DESDE LA CREACIÓN}

La creación del Comité de las Regiones, si bien supuso — como en otro lugar he escrito ${ }^{8}$ - la institucionalización de la cuestión regional, no satisfizo las aspiraciones de las entidades subestatales (llámense Regiones, Länder o Comunidades Autónomas) de la Unión Europea que ansiaban verse reconocidas en una importante posición en el entramado institucional comunitario. Por ello, por muchos, fue considerado «una primera respuesta a la integración de las regiones y de los poderes locales en el proceso de construcción europea» ${ }^{9}$, «el embrión y punto de partida de esa futura participación institucional de las Regiones en la vida de la Unión» ${ }^{10}$ y no una meta, un punto de llegada.

\section{La reforma de Ámsterdam}

Con ocasión de la primera reforma del Derecho originario que habría de tener lugar después del Tratado por el cual se creó este órgano ${ }^{11}$, el propio Comité de las Regiones elevó al grupo de reflexión constituido para realizar este trabajo ${ }^{12}$ en un Dictamen sobre la reforma del Tratado de la Unión Europea de abril de $1995^{13}$ una serie de pretensiones, que tendían, fundamentalmente, a la potenciación institucional de este órgano.

El Comité de las Regiones, en el papel que ha querido asumir de «guardián o defensor del principio de subsidiariedad», propuso, en primer térmi-

\footnotetext{
${ }^{8}$ Calonge Velázquez, A., «El Comité de las Regiones: la institucionalización de la cuestión regional», REALA, nº 263, 1994.

${ }^{9}$ Véase el Informe de la Comisión de política regional, ordenación del territorio y relaciones con los poderes regionales y locales sobre la participación y la representación de las regiones en la construcción europea: el Comité de las Regiones (PE 200.528/DEF) 3-325/93, o la Resolución del Parlamento Europeo A3-0325/93, de 18 de noviembre de 1993, que considera la creación del Comité de las Regiones «un primer paso que deberá evolucionar y complementarse en la revisión prevista en 1996».

${ }^{10}$ Rojo SAlgado, A., La exigencia de la participación regional en la Unión Europea, CEC, Madrid, 1996, p. 164.

${ }^{11}$ El propio Tratado de Maastricht preveía en su artículo N.2 — de manera que no deja de ser ciertamente sorprendente - la propia reforma del Tratado recién aprobado con un texto del siguiente tenor: «En 1996 se convocará una Conferencia de representantes de los Gobiernos de los Estados miembros para que examine de conformidad con los objetivos establecidos en los artículos A y B de las disposiciones comunes, las disposiciones del presente Tratado para las que se prevea una modificación».

${ }^{12}$ En el Consejo Europeo de Corfú de junio de 1994 se adoptó, entre otros, un acuerdo por el que se creó un grupo de reflexión — que se constituiría el 2 de junio de 1995- para preparar la CIG'96 presidido por el representante del país que ostentaba la presidencia de la Comunidad en ese momento: el español Sr. Westendorp y compuesto por los representantes de los Ministros de Asuntos Exteriores y por el Presidente de la Comisión (que delegó en el Comisario español Marcelino Oreja), en el que participarían asimismo dos representantes del Parlamento Europeo.
}

${ }^{13}$ CdR 136/95, Bruselas, 20-21 de abril de 1995. 


\section{EL COMITÉ DE LAS REGIONES EN EL FUTURO DE EUROPA}

no, la adopción de una serie de medidas que tenían por finalidad su participación en la aplicación de dicho principio. En concreto, pueden señalarse las siguientes:

- Una nueva formulación del artículo 3B TCE que configurara la subsidiariedad no únicamente como un criterio de ejercicio de competencias compartidas entre la Unión y los Estados miembros, sino como un criterio de reparto de competencias entre todos los niveles de gobierno que participan en la Unión Europea.

- El establecimiento de los mecanismos adecuados para poder recurrir ante el Tribunal de Justicia por la vulneración de la subsidiariedad que afecte a los entes regionales y locales.

- El inicio de negociaciones que conduzcan a la delimitación clara de las competencias de la Unión y los Estados miembros.

- En fin, la aplicación en el interior por los Estados miembros del principio de subsidiariedad, en relación a las regiones y entes locales.

En segundo lugar, por lo que se refiere al incremento de la capacidad de actuación de este organismo, el Comité formuló las siguientes propuestas:

- El reconocimiento del Comité de las Regiones como Institución europea, a través de la reforma del artículo 4 TCE, sin poner en tela de juicio su carácter consultivo ni la preeminencia de otras instituciones.

- La obtención de la oportuna capacidad procesal para conseguir una legitimación activa privilegiada en los mismos términos reconocidos al Parlamento Europeo y al Banco Central Europeo en el caso del recurso de nulidad previsto por el artículo 173 TCE, así como en el caso del recurso de inactividad contemplado en el artículo 175 del mismo Tratado.

- La exigencia a sus miembros de un mandato electivo o que respondan directamente ante una asamblea elegida por sufragio universal directo.

- El poder de elaborar su reglamento interno sin necesidad de someterlo a la aprobación del Consejo.

- La ampliación de los supuestos de consulta al conjunto de materias de afectación regional o local, especialmente en materias como agricultura, transportes, política social, investigación y desarrollo tecnológico, cooperación al desarrollo, formación profesional, protección al medio ambiente, industria, energía y protección a los consumidores. El carácter preceptivo de la consulta debería quedar afirmado al menos en los mismos términos previstos para el Comité Económico y Social. 
— La ampliación de sus facultades consultivas al Parlamento Europeo.

- El establecimiento de la obligación de las demás Instituciones (Consejo y Comisión) de motivar su decisión cuando se aparten de las recomendaciones contenidas en los dictámenes del Comité.

- Y, finalmente, el reconocimiento de plena autonomía organizativa y presupuestaria, suprimiendo al efecto el Protocolo $n^{\circ} 16$ del TUE.

Las modificaciones producidas en relación a este órgano fueron escasas y poco relevantes ${ }^{14} \mathrm{o}$, si se prefiere adoptar una posición menos negativa, puede afirmarse que los avances del Tratado de Ámsterdam sólo pueden ser calificados, en el mejor de los casos, de discretos en este punto ${ }^{15}$.

En la fase inicial de las negociaciones de la CIG'96 únicamente se había llegado al acuerdo de suprimir el Protocolo $n^{\circ} 16$, que impedía la autonomía organizativa del Comité, y, también, cierto consenso sobre la posibilidad de ampliar los campos de consulta obligatoria a ciertos ámbitos materiales. Avanzadas las negociaciones, algún paso más se dio en la potenciación institucional del Comité de las Regiones.

Así, en primer lugar, se prohibió para los miembros del Comité la posibilidad de ser simultáneamente miembros del Parlamento Europeo; en segundo término, se suprimió la disposición que obligaba a que el reglamento interno de este órgano fuera objeto de aprobación por parte del Consejo; $\mathrm{y}$, por último, se ampliaron los campos de consulta facultativa («aquellos que afecten a la cooperación transfronteriza»), obligatoria (empleo y cuestiones sociales, medio ambiente, disposiciones de aplicación del Fondo Social Europeo, formación profesional y transportes) y, asimismo, se estableció la posibilidad de que el Comité de las Regiones fuera consultado por el Parlamento Europeo.

Recapitulando y teniendo presentes las reivindicaciones planteadas por el Comité de las Regiones, el Tratado de Ámsterdam sólo dio respuesta a aquéllas que tenían por objeto otorgar la autonomía organizativa y presupuestaria al Comité; las que demandaban una ampliación del campo de consulta y, finalmente, las que requerían el derecho de consulta del Parlamento Europeo ${ }^{16}$.

\footnotetext{
${ }^{14}$ En general, las modificaciones que se introdujeron en el Tratado de Ámsterdam no fueron ni numerosas ni profundas y, quizás, por las expectativas que se fueron levantando durante su proceso de elaboración y que no se vieron cumplidas ha merecido que MANGAS MARTín, Araceli, «El Tratado de Ámsterdam: aspectos generales del pilar comunitario», Gaceta Jurídica de la Comunidad Europea, D29, 1998, lo haya calificado de «políticamente mediocre» o de «mera faena aseada», pp. 21 y 24 , respectivamente.

15 Pérez GonzÁlez, Manuel, «Los otros órganos de la Unión», El Tratado de Ámsterdam. Análisis y comentarios, dir. Marcelino Oreja Aguirre, coord. Francisco Fonseca Morillo, vol.I, McGraw-Hill, Madrid, 1998, p. 555.

${ }^{16}$ Sobre estas reformas, más ampliamente, véase mi trabajo «El Comité de las Regiones en el Tratado de Ámsterdam», Noticias de la Unión Europea, n 186, 2000, pp. 181-188.
} 
Otras muchas aspiraciones, sin embargo, se quedaron sin respuesta: la reformulación del principio de subsidiariedad, su ubicación entre las Instituciones comunitarias y el locus standi ante el Tribunal de Justicia, por citar sólo algunas.

\section{La reforma de Niza}

Si el Comité de las Regiones, en la reforma comunitaria que acabamos de examinar, escribió a la Conferencia intergubernamental «una larguísima carta a los reyes magos» ${ }^{17}$, en la siguiente ocasión: la Conferencia intergubernamental 2000 - ejerciendo el derecho que se había reservado de volver a presentar sus peticiones de una mejor representación de los entes locales y regionales, en el marco de una nueva revisión de los Tratados ${ }^{18}$ - no se quedó atrás, pues vuelve a reiterar, prácticamente, las propuestas que hizo con motivo de la reforma del Tratado de Maastricht y que no fueron atendidas, como acabamos de ver, por los Jefes de Estado o de Gobierno al aprobar el Tratado de Ámsterdam.

El Comité de las Regiones, en su Dictamen sobre «La Conferencia Intergubernamental del $2000 »{ }^{19}$, realizó las propuestas de reforma que a él le podían afectar.

En su papel autoatribuido de «guardián o defensor del principio de subsidiariedad», propuso, en primer término, la modificación del artículo 5 TCE para que, además de los límites de las competencias de la Comunidad y de los Estados miembros, se tome en consideración el estatuto especial de los gobiernos regionales y locales. Esto es, volvió a reclamar que el Tratado comunitario configurara la subsidiariedad no únicamente como un criterio de ejercicio de competencias compartidas entre la Unión y los Estados miembros, sino como un criterio de reparto de competencias entre todos los niveles de gobierno que participan en la Unión Europea.

Asimismo demandó que se incluyera, entre los principios comunes a los Estados miembros que sirven de base a la Unión — como la libertad, la democracia, el respeto de los derechos humanos y de las libertades fundamentales y el Estado de Derecho-, el principio de autonomía local y regional, como expresión de ese nivel de gobierno que existe en la Unión Europea.

\footnotetext{
${ }^{17}$ Martín y PÉREZ NANClaRes, Javier, «La reforma institucional y la cooperación reforzada en el Tratado de Ámsterdam», Cursos de Derecho Internacional de Vitoria-Gasteiz 1998, Tecnos-UPV, MadridBilbao, 1999, p.129

${ }^{18}$ Véase la Resolución del Comité de las Regiones sobre los resultados de la Conferencia Intergubernamental, DOCE n ${ }^{\circ}$ C 64/28, de 27 de febrero de 1998.

${ }^{19}$ DOCE $\mathrm{n}^{\circ} \mathrm{C} 156 / 6$, de 6 de junio de 2000.
} 
En tercer lugar, reclamó que se le reconociera el derecho formal de recurso para la defensa de sus competencias, al tiempo que exigió que este derecho le fuera reconocido también a las regiones con poder legislativo.

Por lo que se refiere al incremento de capacidad de actuación de este organismo, el Comité formuló las siguientes propuestas:

Primero, el reconocimiento del Comité de las Regiones como Institución de la Unión Europea, a través de la reforma del artículo 7 TCE, con el objetivo de establecer en Europa un tercer nivel de gobierno de autoridades locales y regionales.

Segundo, el establecimiento de la obligación de las Instituciones a las que auxilia - Consejo y Comisión- de, en los ámbitos de consulta obligatoria, motivar su decisión cuando se aparten de las recomendaciones contenidas en los dictámenes del Comité. El Parlamento Europeo deberá hacerlo —indicó el dictamen del que damos cuenta- de forma voluntaria.

Por lo que respecta al número de miembros del Comité de las Regiones, como consecuencia de la ampliación, el propio Comité consideró adecuado un número máximo aproximado de 350 miembros, que garantizara el equilibrio en el tamaño de las delegaciones, la representación de las autoridades regionales y locales y la distribución geográfica de la representación de cada Estado miembro.

Y, finalmente, en cuanto a la elección de sus miembros, reiteró el Comité la propuesta de que sean titulares de un mandato electivo en una entidad regional o local, o que respondan directamente ante una asamblea elegida por sufragio universal directo. Además, precisó que en el texto del Tratado se consignara que el miembro el Comité que perdiera la condición requerida para ser nombrado, dejara automáticamente de ser miembro después de un plazo de seis meses, garantizando - los Estados miembros- el nombramiento puntual de un sucesor.

Pues bien, la reforma que se ha operado en el TCE en relación al Comité de las Regiones apenas es perceptible; las modificaciones introducidas son poco relevantes. Como ha manifestado Andrés Sáenz de Santa María, el Tratado de Niza ha hecho suyas las peticiones menores del Comité y le ha negado las de mayor envergadura, dato - sigue escribiendo la autora citadaque no debe ser olvidado a la hora de valorar el papel que la Unión Europea reserva a las regiones ${ }^{20}$.

Ni la inclusión del principio de autonomía local y regional entre los principios comunes a los Estados miembros y que sirven de base a la Unión, ni el derecho formal de recurso ante el Tribunal de Justicia para la defensa de

\footnotetext{
20 Andrés SÁEnZ de Santa María, Paz, «La reforma institucional en el Tratado de Niza», Gaceta Jurídica, $\mathrm{n}^{\circ} 211,2001$, p. 25.
} 
sus competencias, ni la obligación de motivación del Consejo y de la Comisión cuando no sigan los dictámenes del Comité, ni el otorgamiento del estatuto de institución han sido atendidas por los Jefes de Estado o de Gobierno reunidos en Niza para la reforma institucional de cara a la ampliación.

Las modificaciones efectuadas — todas ellas, además, en el artículo 263 TCE - son fundamentalmente tres: la primera, da respuesta a una vieja aspiración del Comité de las Regiones, pues se exige que los miembros del Comité, representantes de los entes regionales o locales, sean titulares de un mandato electoral en un ente regional o local, o que ostenten la responsabilidad política ante una asamblea elegida; la segunda, tiene por objeto el número de miembros que integrarán este Comité, que no excederá de 350 y la tercera, se refiere al procedimiento de nombramiento de los miembros de este órgano, pues serán nombrados por mayoría cualificada del Consejo, mientras que con anterioridad se exigía la unanimidad y, además, su mandato está limitado a la exigencia de ser representante de un ente regional o local o políticamente responsable ante una asamblea elegida por sufragio universal y directo, mientras que con anterioridad los nombrados lo eran a título personal sin estar vinculados por ningún mandato imperativo, ejerciendo sus funciones con absoluta independencia, en interés general de la Comunidad ${ }^{21}$.

\section{LAS REIVINDICACIONES FRENTE AL FUTURO}

Concluido el Consejo Europeo de Niza y tras la firma del Tratado que lleva el nombre de dicha ciudad francesa el día 26 de febrero de 2001, el Comité de las Regiones emitió, como había hecho con motivo de la reforma anterior ${ }^{22}$, una Resolución sobre los «Resultados de la Conferencia Intergubernamental de 2000 y el debate sobre el futuro de la Unión Europea» donde manifestó su opinión sobre los contenidos del nuevo Tratado y, por lo que a nosotros aquí más nos interesa, volvió a explicitar las reformas que, en su opinión, precisa este Comité.

Por lo que se refiere a las modificaciones que afectan a los artículos del Tratado que lo regulan, el Comité se manifestó de manera muy favorable. Así, acogió con satisfacción que la Conferencia Intergubernamental haya tenido en cuenta las demandas formuladas desde hace años en cuanto a la necesidad de que sus miembros sean titulares de un mandato político en la región o municipio de origen, condición previa e imprescindible para la obtención y la continuación de su mandato en el Comité; reconoció que el texto del nuevo Tratado responde a sus propuestas en este sentido. Se congratuló, asimismo,

\footnotetext{
${ }^{21}$ Respecto a la reformas introducidas por el Tratado de Niza, véase, in totum, mi trabajo «El Comité de las Regiones de cara a la ampliación.: Las modificaciones de Niza», Noticias de la Unión Europea, $\mathrm{n}^{\circ} 218,2003, \mathrm{pp} .25-34$.

${ }^{22}$ Véase DOCE $n^{\circ}$ C 64/28, de 27 de febrero de 1998.
} 
de que el futuro nombramiento de los miembros y de sus suplentes sea decidido por el Consejo por mayoría cualificada y esperaba que el procedimiento de nombramiento, sobre todo en el caso de sustitución de un miembro dentro de un mandato, sea mucho más rápido. Y, finalmente, se felicitó porque, de acuerdo con sus propuestas, se haya fijado el número máximo de miembros de este órgano en 350, pero se lamentaba de que, con motivo de la ampliación de la Unión, el reparto de los mandatos según los Estados miembros no se ajuste a su papel político específico, y considera inadecuado que el número de miembros y su reparto entre los Estados miembros se siga haciendo según un esquema paralelo al del Comité Económico y Social ${ }^{23}$.

A continuación, en este Resolución que evalúa los resultados alcanzados en Niza, el Comité de las Regiones reitera con insistencia las exigencias relativas a mejorar su participación en el proceso decisorio de la Comunidad y, así, manifiesta, con carácter general, que el Tratado de Niza es claramente insuficiente con respecto a lo que el Comité en sus dictámenes y resoluciones ha considerado imprescindible para fortalecer la capacidad de ampliación de la Unión, para incrementar su legitimidad democrática, su transparencia y su proximidad al ciudadano y mejorar sosteniblemente la eficacia del proceso decisorio; hace hincapié — especialmente — en su petición de que se le reconozca el derecho de recurso ante el Tribunal de Justicia para garantizar el respeto de sus derechos y, sobre todo, para velar por la aplicación efectiva del principio de subsidiariedad; $y$, por último, reclama un estatuto de institución de pleno derecho de la Unión Europea y no meramente con carácter consultivo, sino con auténtico poder y situada al mismo nivel y rango que las demás Instituciones.

En definitiva, el Comité de las Regiones mantiene todas aquellas reivindicaciones que viene manifestando desde prácticamente su creación y que aún no han sido asumidas por los Jefes de Estado o de Gobierno en las reformas que han llevado a cabo.

\section{LA POSICIÓN SOBRE LA CONVENCIÓN}

Desde el mismo momento que la Cumbre de Niza de diciembre de 2000 clausuró sus sesiones se inició un debate más amplio y profundo sobre el desarrollo futuro de la Unión Europea y, en especial, sobre la mejora de su legitimidad democrática y el incremento de la transparencia y de la proximidad

\footnotetext{
${ }^{23}$ La Declaración número 20, aneja al Tratado de Niza, relativa a la ampliación de la Unión Europea expresa la posición común que los Quince han adoptado en las conferencias de adhesión en lo que se refiere, en este caso, al Comité de las Regiones, que será en una Unión Europea compuesta por veintisiete Estados miembros, la siguiente: Alemania, Reino Unido, Francia e Italia, 24; España y Polonia, 21; Rumania, 15; Países Bajos, Grecia, República Checa, Bélgica, Hungría, Portugal, Suecia, Bulgaria y Austria, 12; Eslovaquia, Dinamarca, Finlandia, Irlanda y Lituania, 9; Letonia, Eslovenia y Estonia, 7; Chipre y Luxemburgo, 6 y Malta 5. El número total de miembros del Comité de las Regiones es, pues, de 344 .
} 
a los ciudadanos al que quedarían asociados todas las instituciones y medios interesados así como los países candidatos, que debería desembocar en una declaración del Consejo Europeo de Bruselas-Laeken en diciembre de 2001.

El Comité de las Regiones, en su afán de no limitarse a ser el órgano auxiliar de carácter consultivo configurado en el Tratado comunitario, apoyó expresamente la intención de los Jefes de Estado y de Gobierno de iniciar de manera inmediata un amplio debate público sobre la futura evolución de la Unión Europea; mostró su satisfacción ante la convocatoria de una nueva Conferencia Intergubernamental y, asimismo que el Consejo Europeo bajo la presidencia belga quisiera aprobar en Laeken una Declaración sobre la preparación y los contenidos de la Conferencia Intergubernamental de 2004; e hizo, en la Resolución ya citada de 4 de abril de 2001, una primera manifestación de sus posiciones sobre las cuestiones de mayor enjundia y que le afectan de manera más directa como la delimitación de competencias entre la Unión Europea y los Estados miembros donde propone que se examine la cuestión de cómo repartir mejor las tareas en los ámbitos políticos más importantes entre la Unión Europea, por una parte, y los Estados miembros, sus regiones y municipios, por otra; o la futura arquitectura de las instituciones europeas donde espera que se revalúe el papel que desempeñan las regiones y los municipios en el proceso de unificación europea y en la aplicación próxima a los ciudadanos de la política europea, de conformidad con el principio de subsidiariedad y, para ello, considera necesario transformar al Comité de las Regiones en una Institución, dotarla de una configuración que pueda responder eficazmente a las funciones encomendadas y otorgarle unas competencias que vayan más allá de una función puramente consultiva, de manera que se convierta en un órgano de coparticipación en el proceso de decisión europea, en el que todos los niveles políticos de los Estados miembros desde los municipios hasta las regiones con competencias legislativas, encuentren su lugar y asuman su corresponsabilidad.

Con anterioridad a la convocatoria de la Convención sobre el futuro de Europa por el Consejo Europeo de Laeken de diciembre de 2001, pero lanzado el debate desde la aprobación del Tratado de Niza, el Comité de las Regiones se pronunció en dos ocasiones respecto del debate que se avecinaba.

En el Memorándum de 3 de octubre de 2001 sobre la participación del Comité de las Regiones en el debate estructurado sobre el futuro de Europa ${ }^{24}$, este órgano aporta argumentos bastantes, en mi opinión, para estar representado en el grupo preparatorio de la próxima Conferencia intergubernamental (aún no se sabía — recuérdese- que se seguiría el método ensayado un año antes con la Convención sobre los Derechos Fundamentales) mediante una delegación representativa que pueda participar en la formulación de propuestas y disponga de pleno derecho de veto.

${ }^{24}$ CDR 325/2001 fin. 
Son cinco las razones esgrimidas por el Comité de las Regiones que justifican su participación de pleno derecho en el debate estructurado sobre el futuro de la Unión:

$1^{\text {a }} \quad$ El nivel subnacional se verá directamente afectado por las reformas que se hagan, sobre todo en lo que se refiere a la delimitación de competencias entre la Unión Europea y los Estados miembros.

$2^{\mathrm{a}} \quad$ El Comité de las Regiones representa el nivel subnacional en toda su diversidad, puesto que es el único órgano comunitario legitimado por los Tratados para representar al conjunto de las regiones y los municipios de los Estados miembros de la Unión.

$3^{\mathrm{a}} \quad$ El Comité de las Regiones refuerza la legitimación democrática del grupo encargado de los trabajos preparatorios en cuanto la inmensa mayoría de sus miembros ha recibido ya un mandato democrático de los electores de sus municipios y regiones y por tanto dispone de una legitimación democrática tan firme y directa - asimilable, en consecuencia - con los miembros de los parlamentos nacionales y del Parlamento Europeo.

$4^{\mathrm{a}} \quad$ El Comité de las Regiones puede aumentar la transparencia de los trabajos, ya que sus miembros tienen contacto directo y permanente sobre el terreno con los ciudadanos y, desde esta posición, puede contribuir con ahínco a que éstos estén informados y participen de manera activa en el debate sobre el futuro de Europa.

$5^{\text {a }} \quad$ El Comité de las Regiones puede aportar una contribución sustancial gracias a su experiencia, sobre todo en lo que se refiere a una mejor delimitación de las competencias que afecten directamente al nivel subnacional, que es el que él representa, pero también en el debate sobre la arquitectura institucional y el proceso decisorio de formación de voluntades en el seno de la Unión.

La segunda vez que se pronunció fue con ocasión de su Resolución de 20 de noviembre de 2001 sobre «La preparación del Consejo Europeo de Laeken y el desarrollo de la Unión Europea en el marco de la próxima Conferencia Intergubernamental de 2004» ${ }^{25}$.

En esta Resolución, después de recordar su satisfacción por la convocatoria de una Conferencia intergubernamental para el año 2004, así como por el objetivo de comenzar a preparar la misma por medio de un debate amplio y profundo sobre el futuro de la Unión Europea, se manifiesta sobre su contenido, llegando a considerar oportuno - en primer término- que se amplíen las cuatro áreas temáticas definidas en el Tratado de Niza integrándolas en problemáticas más abiertas y considera que deberían incluirse, entre otros, temas como la manera de fomentar un modelo de desarrollo sostenible y

${ }^{25}$ CDR 104/2001 fin. 
solidario que permita profundizar en el objetivo de la cohesión económica, social y territorial, así como avanzar hacia un modelo más participativo de gobernanza; y analizando, después, cada uno de los temas de debate propuestos en la conocida Declaración $n^{\circ}$ 23, aneja al Acta Final del Tratado de Niza, sobre el futuro de la Unión Europea.

Así, en relación a la delimitación de competencias entre la Unión Europea y los Estados miembros considera necesario aclarar qué cometidos deben y pueden llevar a cabo juntos los miembros de una Unión enormemente ampliada y solicita que el Tratado incluya una descripción clara de los cometidos de la Unión en aras de una mayor transparencia sobre los ámbitos cuya responsabilidad política recae en la Unión Europea. Ahora bien, no se pronuncia ni respecto a la distribución concreta de ámbito de actuación, ni siquiera, sobre cuál puede ser el mejor sistema de distribución de misiones entre la Unión Europea y los Estados miembros.

Respecto de la Carta de los derechos fundamentales de la Unión Europea se limita a abogar por su integración en los Tratados para dejar claro que la Unión Europea constituye una comunidad de valores, aunque no se adentra, en absoluto, en toda la problemática que supondría dicha integración en un texto jurídico de carácter vinculante.

En relación al papel de los parlamentos nacionales en la arquitectura europea, aboga por reforzar su participación informal en la preparación de las modificaciones del Tratado y en la elaboración de los proyectos en relación con la Unión Europea, con el fin de fortalecer el debate sobre política europea en los Estados miembros; pero se muestra contrario a la creación de una nueva institución formada por representantes de los parlamentos nacionales, pues no la considera ni conveniente ni necesaria.

En fin, por lo que se refiere a la simplificación de los Tratados se pronuncia por una iniciativa constitucional que mejore su claridad e inteligibilidad. Por medio de esta iniciativa se deberían agrupar todos los Tratados en un texto único formado por una parte general y una parte dedicada a reglamentar las políticas de la Unión, esto es, se pronuncia por la propuesta que en su día elaboró el Instituto Europeo de Florencia ${ }^{26}$, aunque rechaza un procedimiento de modificación del Tratado «autónomo y a nivel comunitario», especialmente por lo que se refiere a la propuesta de suprimir la obligación de ratificación por los parlamentos nacionales.

Aprovecha, por último, esta Resolución para volver a expresar sus aspiraciones y manifestar nuevas reivindicaciones, en cuanto Comité, en la reforma del Derecho originario que se avecina, y, en este sentido:

\footnotetext{
${ }^{26}$ La propuesta de Tratado constitucional elaborada por el Instituto citado en el texto puede verse en el sitio internet: http://www.iue.it (consultado el día 3 de abril de 2003).
} 
- pide el reconocimiento de su legitimidad institucional, de su estatuto especial como representante de los actores locales y regionales, y de su papel político en el debate sobre el futuro de la Unión, así como que se le conceda expresamente el derecho a participar como miembro de pleno derecho en los trabajos de la Convención encargada de preparar la Conferencia Intergubernamental de 2004;

- propone que se reconsidere del papel que el Comité de las Regiones y los entes locales y regionales están llamados a desempeñar en la futura arquitectura europea de acuerdo con el principio de subsidiariedad;

- defiende que se concedan al Comité amplias competencias para que éste pueda reforzar su influencia en el proceso decisorio europeo, así como que estas competencias deberían ir más allá de la función meramente consultiva;

- considera necesario que se otorgue al Comité el estatuto de institución, así como el derecho de recurso ante el Tribunal de Justicia de las Comunidades Europeas para defender sus derechos o en caso de que se contravenga el principio de subsidiariedad;

- pide que la Comisión Europea y el Consejo estén obligados a explicar de qué forma han tenido en cuenta los dictámenes del Comité de las Regiones o por qué no los han tenido en cuenta;

- insta a que el Comité de las Regiones como tal sea habilitado para interpelar oralmente y por escrito a la Comisión Europea;

- opina que las regiones y los entes municipales deberían tener una mayor participación en el proceso decisorio europeo en el marco de las delegaciones nacionales del Consejo.

En fin, el Comité de las Regiones escribe, de nuevo, en este apartado una «larga carta a los reyes magos» dirigida a la Convención que habrá de preparar la Conferencia Intergubernamental 2004 con la intención de obtener alguna de sus viejas aspiraciones y situarse en la arquitectura institucional europea con un mayor peso político que el que tiene en la actualidad.

\section{LA CONTRIBUCIÓN A LA CONVENCIÓN SOBRE EL FUTURO DE EUROPA}

En su $45^{\circ}$ Pleno, celebrado los días 3 y 4 de julio de 2002 (sesión del 4 de julio), el Comité de las Regiones aprobó por unanimidad su contribución a la Convención que, desde el 28 de febrero de 2002, se encargó de realizar los trabajos preparatorios de la Conferencia intergubernamental $2004{ }^{27}$.

${ }^{27}$ Contribución del Comité de las Regiones a la Convención Europea, Bruselas, 8 de julio de 2002, CDR 127/2002 fin. 


\section{EL COMITÉ DE LAS REGIONES EN EL FUTURO DE EUROPA}

En el presente epígrafe es nuestro propósito dar cuenta y analizar esta contribución que se presenta como la manifestación a todos los miembros de la Convención de «las principales aspiraciones del Comité de las Regiones en relación con el futuro de la Unión Europea».

Antes de pasar a la exposición de sus aspiraciones de cara a la futura Unión, el Comité de las Regiones quiere dejar claro que desea que el proceso de reforma de la Unión Europea emprendido contribuya a un mayor acercamiento entre las instituciones europeas y los ciudadanos, y a una mayor adhesión de los ciudadanos al proyecto europeo, abogando, en consecuencia, por una mayor legitimidad democrática de la Unión, como viene haciendo desde hace años ${ }^{28}$. Asimismo, muestra su posición muy favorable en relación con la convocatoria de la Convención, ya que - a su juicio - supone un nuevo método más democrático de reforma del Tratado y, por ello, insta a la Conferencia Intergubernamental 2004 a que siga las propuestas que formule la Convención.

En cuanto a las pretensiones de reforma es nuestra intención exponerlas y analizarlas en torno a tres ejes de acción: en primer término, aquéllas que son generales; en segundo lugar, las que tienen que ver con las misiones y competencias de la Unión y, por último, las que tienen por objetivo mayor democracia, transparencia y eficiencia en la Unión Europea.

En el primer grupo de deseos de reformas, el Comité de las Regiones se pronuncia a favor de la incorporación de la Carta de Derechos Fundamentales de la Unión Europea al nuevo marco constitucional de la Unión, dando así cuerpo a la ciudadanía europea, término - como es sabido - acuñado en el artículo 8 del Tratado de Maastricht, pero escasa e insuficientemente desarrollado hasta el momento presente. La inserción de la Carta de Derechos Fundamentales en el Tratado constitucional a fin de que se la dote de fuerza jurídica vinculante será una contribución esencial, que permitirá que todo nacional de un Estado miembro de la Unión identifique su ciudadanía europea específica como portadora de nuevos derechos y expresión de pertenencia a esta nueva colectividad que es la Unión Europea.

El Comité de las Regiones, pues, da una respuesta afirmativa a uno de los puntos que, a tenor de lo dispuesto en la varias veces mencionada declaración $\mathrm{n}^{\circ} 23$ aneja al Tratado de Niza, conformaría el orden del día de la próxima Conferencia intergubernamental, aunque sin entrar, pues es un papel que nunca ha querido, en los aspectos técnicos de ésta ni de ninguna cuestión ${ }^{29}$.

\footnotetext{
28 Véanse el Informe sobre la Proximidad (CDR 436/2000 fin) y la Declaración final «Nuevas formas de gobierno en Europa: hacia más democracia y proximidad (CDR 379/2000 fin).

29 En otro lugar hemos escrito que el Comité de las Regiones no ha querido quedar confinado a ser un mero órgano consultivo de carácter técnico, sino que desde su constitución ha buscado desempeñar un papel político en el sistema institucional comunitario, lo que le ha conferido en el ejercicio de la actividad desplegada una naturaleza política. Véase CALonge VelázQuez, Antonio y SANZ Rubiales, Iñigo, El Comité de las Regiones. Análisis de una ¿futura institución?, Comares, Granada, 2000, p. 49.
} 
Otro pronunciamiento que realiza este órgano representativo de las entidades regionales y locales es a favor del reforzamiento en la aplicación del principio de subsidiariedad, garante de la base democrática de las instituciones de la Unión y de la puesta en marcha de la ciudadanía europea, reafirmando así el papel de «guardián de la subsidiariedad», que se autoatribuyó desde los inicios de su funcionamiento Y, más allá todavía, reclama en el nuevo marco constitucional de la Unión Europea la incorporación de la Carta Europea de Autonomía Local firmada el 15 de octubre de 1985, aprobada por el Consejo de Europa, con objeto de construir una Europa basada en los principios de democracia y descentralización, así como inspirarse en el Proyecto de Carta Europea de Autonomía Regional, también del Consejo de Europa. Es decir, el Comité de las Regiones pretende se incorpore en la próxima reforma de los Tratados, entre los principios de la Unión, los de autonomía local y regional con la finalidad de que se produzca un reconocimiento en el nivel del Derecho originario de la realidad y el peso político que tienen los municipios y regiones en el proceso de construcción europea.

Asimismo, el Comité de las Regiones considera necesaria una simplificación del funcionamiento de la Unión que incluya una definición clara de la personalidad jurídica de la Unión Europa de la que carece en la actualidad y la simplificación, también, de su estructura actual basada en tres pilares, estructura que conlleva una heterogeneidad jurídica que menoscaba su capacidad de acción.

Por último, reclama que se le reconozca su legitimidad exclusiva como interlocutor institucional de los entes locales y regionales en el marco de la Unión y el reforzamiento de su papel institucional y sus funciones atribuyéndole el status de institución con el mismo rango que el resto de las instituciones comunitarias previstas en el artículo 7 TCE y reforzando su participación en los procedimientos de toma de decisiones, esto es, no quedando relegado al simple papel que tiene en la actualidad de órgano meramente consultivo y sin que sus dictámenes deban ser seguidos por las instituciones dotadas de poder decisional.

Como vemos, pues, hasta ahora el Comité de las Regiones no se separa del guión que se marcó ya con ocasión de la primera reforma de los Tratados que conoció. Si acaso, como puso de manifiesto en sus reivindicaciones ante la Cumbre de Niza, lo ha ampliado solicitando una mayor presencia institucional en el proceso de integración europea.

En relación a las misiones y competencias de la Unión ${ }^{30}$, el Comité de las Regiones sostiene que, en aras a la proximidad a los ciudadanos, es ne-

\footnotetext{
${ }^{30}$ Una primera contribución de este órgano a la Convención versó, precisamente, sobre esta cuestión, que es, sin duda, la que más puede afectar a los entes a los que representa. Véase su Dictamen sobre el «Proyecto de informe del Parlamento Europeo sobre la delimitación de competencias entre la Unión Europea y los Estados miembros», CDR 127/2002 fin.
} 
cesaria una mayor transparencia y responsabilidad en el futuro sobre los ámbitos cuya competencia recae en la Unión Europea y, asimismo, se muestra contrario al establecimiento en el futuro Tratado constitucional de listas tasadas de competencias y entiende que la Unión ha de contar con todas aquellas competencias que resulten necesarias para el logro de sus objetivos y los instrumentos adecuados para desempeñar sus misiones.

Así, pues, el Comité de las Regiones entiende que es precisa una delimitación de competencias más transparente en el seno de la Unión, ya que — manifiesta - la adhesión de los ciudadanos a la Unión sólo puede lograrse si la construcción europea se sigue realizando en un marco de transparencia por lo que respecta a las competencias y las responsabilidades. Conviene, por tanto, determinar claramente dónde se sitúa la responsabilidad política y dónde se ejerce el control democrático. En este sentido, el Comité de las Regiones comparte con el Parlamento Europeo ${ }^{31}$ la distinción entre competencias propias o exclusivas, compartidas y complementarias de la Unión, así como que la mayor parte de éstas son compartidas, lo que exige la articulación de mecanismos de responsabilidad.

Según el Comité de las Regiones, esta operación de mejor definición y distribución de competencias debe basarse en los principios de subsidiariedad, proporcionalidad y proximidad.

Por lo que respecta al principio de subsidiariedad, el Comité de las Regiones entiende que, en virtud del mismo, el nivel europeo debe dejar paso prioritariamente a los otros niveles de acción — los Estados miembros y sus entes regionales y locales- en los ámbitos que no sean de su competencia exclusiva. Y, además, recuerda que ha venido reclamando, desde sus inicios, que se le atribuya expresamente en los Tratados la función de velar por la observancia de este principio, lo que vuelve a hacer en este documento entendiendo que la institución que representa a las instancias de gobierno más cercanas a los ciudadanos tenga un papel específico en velar por el respeto del señalado principio.

En relación con la cuestión del mantenimiento del artículo 308 TCE en su formulación actual, el Comité de las Regiones manifiesta que considera que la Unión Europea debe poder seguir reaccionando con flexibilidad ante retos cada vez mayores y desea preservar el mecanismo de la integración, lo que implica que se debe garantizar una posibilidad de evolución futura en la definición de las misiones de la Unión y la distribución de sus competencias.

En cuanto a la distribución de competencias, el Comité de las Regiones considera que deben reforzarse los objetivos políticos que figuran en el artículo 2 del Tratado de la Unión. Se pronuncia a favor de:

\footnotetext{
31 Véase la Resolución del Parlamento Europeo sobre la delimitación de competencias entre la Unión Europea y los Estados miembros [2001/2024 (INI)].
} 
- la adopción de una auténtica política exterior y de seguridad común, con medidas como la atribución a la Unión de competencias exclusivas en este ámbito, para conferirle un papel más importante en la escena internacional;

- la realización de un espacio de libertad, seguridad y justicia para los ciudadanos europeos, presidido por la Carta de Derechos Fundamentales como instrumento básico de garantía de los derechos;

- la consolidación del modelo social y económico europeo, en el que la cohesión social y territorial, el alto nivel de protección social y la calidad de vida, lejos de ser incompatible con el progreso económico, constituyen la condición de competitividad de cada territorio;

- una política de desarrollo sostenible en el contexto de una mejor coordinación de las políticas medioambiental, social y económica;

El Comité de las Regiones desea, por otra parte, que la cohesión económica, social y territorial se reconozca claramente como una de las misiones prioritarias de la Unión Europea. Para reforzar la coherencia de las disposiciones en el marco jurídico de la Unión, aspira a que la cohesión territorial figure entre los principios fundamentales del Tratado, al igual que la cohesión económica y social. Y ello porque entiende que la ampliación va a aumentar la heterogeneidad de la Unión y a acentuar las disparidades entre las regiones, lo que exige un mayor esfuerzo en este ámbito para garantizar los objetivos de integración política y económica que se fijó la Unión Europea.

Por último, entiende que en este marco es necesaria una sistematización más precisa de los instrumentos normativos de la Unión Europea, que comprenda el establecimiento de una jerarquía normativa y considera que la utilización de normas marco y directivas como instrumentos jurídicos más idóneos deberá prevalecer sobre las regulaciones más detalladas, reservadas para cuando sean estrictamente necesarias para la consecución del objetivo.

Así, pues, el Comité de las Regiones en este segundo grupo de reclamaciones se pronuncia por una mejor y más clara definición de las misiones - a las que añade la cohesión territorial - que corresponden a la Unión Europea, así como una delimitación más precisa de las competencias entre la Unión y los Estados miembros basada en un sistema que tenga como principales principios inspiradores los de subsidiariedad - del que reclama un mayor protagonismo como órgano representante de las instancias más próximas al ciudadanos-, proporcionalidad y proximidad.

Por lo que respecta al tercer grupo de peticiones presentadas en esta Contribución - agrupadas en torno a la idea de «más democracia, transparencia y eficiencia en la Unión Europea»—, el Comité de las Regiones, como úni- 
ca expresión institucional de los entes locales y regionales en la Unión Europea, exige inexcusablemente que se potencie la participación de estos entes en los procesos de toma de decisiones y que se aumente su participación en la preparación y aplicación de las políticas europeas, lo que requiere a su vez que se refuerce la posición institucional y las funciones del propio Comité como cauce para hacer efectiva esta participación. Esta mayor participación debe ser de dos formas: por una parte, la consulta sistemática en el periodo pre-legislativo y, por otra, mediante el refuerzo de su papel en el proceso de toma de decisiones.

Pues bien, el Comité de las Regiones, para obtener ese reforzamiento del papel de los entes regionales y locales que otorgue mayor legitimidad democrática a la Unión Europea, demanda irrenunciablemente:

1. El reconocimiento del estatus de institución

2. Legitimación activa ante el Tribunal de Justicia, al menos en defensa de sus prerrogativas y del principio de subsidiariedad.

3. Fortalecimiento de las funciones del Comité.

En cuanto a sus funciones, el Comité ha manifestado - y vuelve a hacerlo en el presente documento- su deseo de que le sean asignadas funciones que vayan más allá de las meramente consultivas que ostenta en la actualidad. Para ello, debería otorgarse al Comité un derecho de veto suspensivo en algunos de los casos de consulta obligatoria y, asimismo, la facultad de poder asistir al procedimiento de codecisión entre el Consejo, el Parlamento Europeo y la Comisión para los diez casos de consulta obligatoria previstos en el Tratado.

En relación con sus funciones consultivas, el Comité de las Regiones plantea las siguientes modificaciones que podrán introducirse con la finalidad de que resulten fortalecidas:

- La falta de consulta preceptiva al Comité o la adopción de un instrumento legislativo dentro de sus ámbitos de consulta sin que este órgano haya dictaminado debería ir acompañada de un mecanismo que hiciera efectiva en la práctica la consecuencia jurídica de tal omisión. En especial, el Comité de las Regiones ha de ostentar legitimación activa en defensa de sus prerrogativas, lo que le habilitaría a instar ante el Tribunal de Justicia la nulidad de los actos comunitarios adoptados sin la consulta preceptiva del Comité.

- El Comité de las Regiones solicita realizar esta función consultiva mediante la obligación por parte de las instituciones que adopten la norma de justificar la desviación respecto de la opinión del Comité. Esta obligación debe extenderse a todas las materias en que la consulta sea preceptiva. 
- Conviene, finalmente, ampliar la lista de materias en que es preceptiva la consulta al Comité, hasta extenderla a todas aquellas materias en que gozan de competencias los entes locales y regionales, tales como, por ejemplo, agricultura e investigación y desarrollo tecnológico.

Por lo que se refiere a sus restantes funciones, el Comité de las Regiones considera que es conveniente que ostente:

- La posibilidad de interpelar por escrito y oralmente a la Comisión Europea.

- Las funciones codecisorias en un número determinado y pertinente de ámbitos, habida cuenta el objetivo fijado de simplificación de procedimientos de adopción de decisiones, pero que incluya, en cualquier caso, los programas de fomento de la cooperación transfronteriza (especialmente, iniciativas comunitarias como Interreg).

Como vemos, pues, en este apartado todas las reclamaciones que presenta tienen como principal destinatario el mismo Comité. Vuelven a mezclarse, como con ocasión de la última reforma de los Tratados, reivindicaciones viejas con nuevas aspiraciones. El Comité de las Regiones no se considera ya sólo, como desde sus inicios, «guardián de la subsidiariedad», sino que también entiende que es y debe ser el único representante de los entes locales y regionales en el proceso de construcción europea, lo que le permite solicitar el estatus de institución al mismo nivel que el resto de instituciones comunitarias; pedir el fortalecimiento de sus funciones extendiéndolas más allá de las meramente consultivas; reclamar la ampliación de sus ámbitos de consulta; reivindicar la obtención de cierta capacidad de control político sobre la Comisión, al menos; y, en fin, pedir la capacidad de codecidir con las instituciones dotadas de poder legislativo en un «número determinado y pertinente de ámbitos» que deja, precisamente, sin determinar y al albur de la decisión de los que tienen la capacidad de modificar los Tratados.

El Comité de las Regiones ha vuelto, pues, a escribir su particular y abultada - en mi opinión- «carta a los reyes magos» reclamando a la Convención sobre el futuro de Europa un papel que, de momento, los Jefes de Estado y de Gobierno no son proclives a otorgarle, pero es que, además, no hay una sensibilidad en las instancias europeas en relación con las reivindicaciones que plantea el Comité de las Regiones y ello por la sencilla razón de que la Unión Europea que se construye no es, ni mucho menos, una agrupación de regiones y entes locales, sino ante todo y sobre todo una unión de Estados donde la heterogenidad regional y local es muy vasta. 


\section{LOS RESULTADOS: EL TRATADO POR EL QUE SE ESTABLECE UNA CONSTITUCIÓN PARA EUROPA}

\section{Idéntica regulación: naturaleza jurídica, composición y competencias}

Pese a que, como - de manera acertada - haya observado MANGAS MARTín ${ }^{32}$, en el Tratado por el que se establece una Constitución para Europa de 28 de octubre de 2004, el Comité de las Regiones es citado y regulado antes que el Comité Económico y Social, en reconocimiento a la importancia política de su composición y, asimismo, que este Tratado menciona más que ningún otro a las Regiones, lo cierto es que, según he escrito ya en otro lugar ${ }^{33}$, la llamada Constitución Europea no supone ningún avance para este Comité ni para las aspiraciones de las Regiones que quieren ver incrementada su influencia y su presencia en la Unión Europea, aunque tampoco debe añadirse - ningún paso atrás.

Al Comité de las Regiones la Constitución para Europa dedica, en la Parte I, el artículo 32, que se encuentra en el Capítulo Segundo, bajo la rúbrica de «Otras instituciones y órganos consultivos de la Unión», del Título IV; y los artículos III-386 a III-388 del Título VI que regula el «Funcionamiento de la Unión». Pues bien, estos preceptos - con algunas pequeñas novedadesson idénticos a los vigentes artículos 7.2 y 263 a 265 TCE. Demostremos lo correcto de esta afirmación sobre la base de tres aspectos importantes en la vida y actividad de este órgano como son su naturaleza, su composición y sus competencias.

\section{Naturaleza jurídica}

El Comité de las Regiones sigue siendo un órgano secundario, privado del status y rango de institución comunitaria, que tiene como función asistir al Consejo, a la Comisión y, ahora, también, al Parlamento Europeo en todos aquellos casos previstos en el Tratado. Sigue siendo, pues, un órgano de naturaleza asesora - con funciones consultivas - integrado por representantes de las entidades subestatales de la Unión Europea.

Así se desprende, con toda claridad, del tenor literal de los artículos I-32.1 cuando dice que tanto este órgano como el CES «ejercerán funciones consultivas»; y III-388 cuando manifiesta que «el Comité de las Regiones será consultado por el Parlamento Europeo, por el Consejo o por la Comisión, en los casos previstos en la Constitución...».

\footnotetext{
32 La Constitución Europea, Iustel, Madrid, 2005, pp. 130 y 133.

33 «España y el Comité de las Regiones», Cuadernos Europeos de Deusto, n 32, 2005, p. 39.
} 
El Comité de las Regiones sigue siendo ese tipo de órgano de segundo orden cuya tarea es expresar su parecer cuando, atendiendo al ordenamiento, pueden o deben ser consultados. No tiene función legislativa, pues ni puede elaborar normas (más allá de su propio reglamento interno), ni tiene capacidad de iniciativa legislativa, y carece del poder de veto o de enmienda a proposiciones de normas comunitarias, aunque sí interviene en los procedimientos de adopción de decisiones y como parte esencial expresando su opinión con respecto a la propuesta objeto de debate.

Por otra parte, su actividad no se circunscribe a la emisión de dictámenes, sino que, desde el principio, se ha pronunciado a través de otras modalidades no contempladas en los Tratados comunitarios y que, más tarde, fueron recogidas en su reglamento interno ${ }^{34}$, de lo que parece desprender cierto carácter o naturaleza parlamentaria, que es, por otra parte, lo que lleva buscando, desde sus orígenes, para convertirse a la postre en la Cámara territorial de la Unión Europea.

Además, sigue teniendo una naturaleza política, lo que se deduce tanto de la composición que alberga como de la actividad que despliega. El hecho de estar compuesto por representantes titulares de un mandato electoral en un ente regional o local o tener responsabilidad política ante una asamblea de elegidos, como ya señalara el artículo 263 TCE en su versión de Niza y, ahora, reitera el artículo I-32.2 de la Constitución Europea, lo convierte en una instancia política y no en un órgano técnico especializado del Consejo, de la Comisión y del Parlamento Europeo. Y, por otra parte, al pronunciarse sobre tan diversos campos y materias y con el sentido que lo viene haciendo se presenta, también, con un claro y evidente carácter o naturaleza política, pues a través de la voz de sus miembros (Presidentes de los Länder alemanes y austriacos, de las Comunidades Autónomas españolas, de las Regiones belgas o italianas y responsables de las principales urbes europeas o representantes de las más importantes organizaciones de las entidades locales) se hacen oír las voces de las Administraciones públicas más próximas a los ciudadanos y, en suma, la voz de éstos.

Y, por último, sigue siendo un órgano de naturaleza híbrida, ya que está integrado - de manera obligatoria, como conocemos- no sólo por representantes de los entes regionales, sino también de los locales. El artículo I-32.2 de la Constitución Europea no hace sino expresar lo que ya manifestaba el artículo 263 TCE, esto es, que el Comité de las Regiones «estará compuesto por representantes de los entes regionales y locales».

\footnotetext{
${ }^{34}$ El reglamento interno, aprobado por Decisión del Consejo de 18 de noviembre de 1999 (DOCE ${ }^{\circ}$ $18 / 25$, de 22 de enero de 2000), estableció, en su artículo 42 que las resoluciones «guardarán relación con el ámbito de la Unión Europea, tendrán por objeto intereses importantes de los entes regionales y locales y serán de actualidad» (apartado $1^{\circ}$ ). Por su parte, el vigente reglamento interno contempla los dictámenes, los informes y las resoluciones (artículos 38-43), si bien ya no explica qué son estas últimas (Decisión de 18 de noviembre de 1999, versión de 11 de febrero de 2004, DOUE n L 175/1 de 10 de mayo de 2004).
} 
En suma, tras la aprobación del Tratado por el que se establece una Constitución para Europa, la misma naturaleza que predicábamos de este órgano a poco de su creación ${ }^{35}$ la seguimos afirmando, lo que demuestra que ningún paso adelante se ha producido, nada nuevo se ha incorporado.

\section{Composición}

El artículo III-386, al igual que hace el artículo 258 TCE, comienza diciendo que «el número de miembros del Comité de las Regiones no excederá de trescientos cincuenta», pero, después, en lugar de llevar a cabo una distribución de los asientos entre los Estados miembros, remite a una decisión europea, adoptada por unanimidad por el Consejo, a propuesta de la Comisión, el establecimiento de la composición del Comité. No debe olvidarse que la Declaración núm. 20, aneja al Tratado de Niza, relativa a la ampliación de la Unión Europea, expresó la posición común que los Estados miembros adoptaron en las conferencias de adhesión en lo que se refiere, en nuestro caso, a la composición del órgano que estamos estudiando, que será, en una Unión Europea a veintisiete, la siguiente:

$\begin{array}{lllr}\text { ALEMANIA } & 24 & \text { BULGARIA } & 12 \\ \text { REINO UNIDO } & 24 & \text { AUSTRIA } & 12 \\ \text { FRANCIA } & 24 & \text { ESLOVAQUIA } & 9 \\ \text { ITALIA } & 24 & \text { DINAMARCA } & 9 \\ \text { ESPAÑA } & 21 & \text { FINLANDIA } & 9 \\ \text { POLONIA } & 21 & \text { IRLANDA } & 9 \\ \text { RUMANIA } & 15 & \text { LITUANIA } & 9 \\ \text { PAÍSES BAJOS } & 12 & \text { LETONIA } & 7 \\ \text { GRECIA } & 12 & \text { ESLOVENIA } & 7 \\ \text { REPÚBLICA CHECA } & 12 & \text { ESTONIA } & 7 \\ \text { BÉLGICA } & 12 & \text { CHIPRE } & 6 \\ \text { HUNGRÍA } & 12 & \text { LUXEMBURGO } & 6 \\ \text { PORTUGAL } & 12 & \text { MALTA } & 5 \\ \text { SUECIA } & 12 & \text { TOTAL } & \mathbf{3 4 4}\end{array}$

${ }^{35}$ Vid. el trabajo escrito en colaboración con SAnZ Rubiales, Iñigo, El Comité de las Regiones, cit., pp. 43-52. 
Además, amplía el mandato del Comité de las Regiones de cuatro a cinco años para vincularlo a las Instituciones para las que emite sus opiniones, es decir, a la Comisión y al Parlamento Europeo que, como sabemos, son las que tienen un período limitado para el ejercicio de sus funciones de cinco años de duración. Y, asimismo, con la finalidad de reafirmar la independencia de sus miembros (los miembros del Comité no estarán vinculados por ningún mandato imperativo y ejercerán sus funciones con absoluta independencia en interés general de la Comunidad), prohíbe que puedan ostentar simultáneamente la condición de miembro del Parlamento Europeo.

Por lo demás, su mandato será renovable y concluirá automáticamente cuando dejen de ostentar la condición por la que fueron elegidos, esto es, cuando finalice su cargo representativo regional o local, siendo sustituidos, por el mismo procedimiento, por el período restante de dicho mandato.

El Consejo, mediante decisión europea, adoptará, la lista de miembros y suplentes establecida de conformidad con las propuestas presentadas por cada Estado miembro.

En fin, como se puede observar, salvo el incremento en un año del período de mandato de este órgano y la prohibición expresa de ostentar de manera simultánea la condición de miembro del Parlamento Europeo y de miembro del Comité, ninguna novedad introduce el Tratado Constitución, por lo que, también, en este punto es cierta la afirmación que intentamos demostrar.

\section{Competencias}

El Comité de las Regiones será consultado por las instituciones a las que asiste en los casos previstos por la Constitución y en cualesquiera otros en que una de dichas instituciones lo estime oportuno, en particular aquellos que afecten a la cooperación transfronteriza. Este es, más o menos, el tenor literal del artículo 265 TCE que se compadece — prácticamente por igual- con el artículo III-388 de la Constitución Europea.

Las competencias de que dispone este órgano se han ido decantando a lo largo de su corta historia y, desde luego, podemos afirmar que se trata de un proceso dinámico que, como la propia Unión Europea, no ha concluido. En sus inicios, cinco eran las materias en que la consulta al Comité era obligatoria: educación, formación profesional y juventud (artículo 126.4); cultura (artículo 128.5); salud pública (artículo 129.4); en el sector de redes transeuropeas, las orientaciones relativas a su establecimiento y las demás medidas serán adoptadas después de haber consultado al Comité Económico y Social y al Comité de las Regiones (artículo 129 D); en materia de cohesión económico y social, las acciones específicas que pueda adoptar el Consejo por unanimidad (artículo 130 B), la definición de las misiones, los objetivos prio- 
ritarios, la organización de los fondos estructurales, las reglas generales aplicables a los fondos y disposiciones necesarias para asegurar su eficacia, así como la coordinación de los fondos entre ellos y con los otros instrumentos financieros existentes, la institución del fondo de cohesión y, finalmente, las decisiones de aplicación relativas al Fondo Europeo de Desarrollo Regional (FEDER) (artículos 130 D y 130 E).

El Tratado preveía, además, otros supuestos de consulta, pero no obligatoria. Así, el primer párrafo del artículo 198 C TCE indicaba que el Consejo o la Comisión podrían consultar al Comité de las Regiones en cualquier caso que lo estimara oportuno, lo que se sigue manteniendo, como hemos tenido ocasión de comprobar; y el párrafo tercero del mismo precepto establecía -igual que hoy lo hacen los artículos 265 TCE y III-388 Constitución Europea- que «Cuando el Comité Económico y Social sea consultado en aplicación del artículo 198, el Consejo o la Comisión informarán al Comité de las Regiones de esta solicitud de dictamen. El Comité de las Regiones podrá emitir un dictamen al respecto cuando estime que hay en juego intereses regionales específicos». Se trata, pues, de dictámenes facultativos y, en cierto modo, subordinados a los del Comité Económico y Social.

Finalmente, el párrafo cuarto de ese mismo artículo 198 C TCE confería al Comité de las Regiones el derecho a emitir dictámenes por propia iniciativa cuando lo considerara conveniente, que es lo mismo que dicen hoy los reiterados preceptos del TCE y de la Constitución Europea.

El Tratado de Ámsterdam amplió el ámbito competencial de consulta de este órgano. En primer término, por lo que respecta a los asuntos que pueden ser sometidos a consulta, a propuesta de España, se incluyó una referencia expresa a «aquellos que afecten a la cooperación transfronteriza». Y, en relación a los ámbitos de consulta preceptiva se extendió a las materias siguientes: empleo y cuestiones sociales (artículo 129), medio ambiente (artículos 161 y 162), disposiciones de aplicación del Fondo Social Europeo (FSE) (artículo 148), formación profesional (artículo 137) y transportes (artículo 71).

La siguiente revisión del Derecho originario, que fue llevada a cabo por el Tratado de Niza, introdujo, por lo que a nuestro órgano se refiere, modificaciones poco relevantes. El ámbito competencial de este órgano se mantuvo inalterado y las incorporaciones se limitaron a algunas cuestiones relativas al nombramiento y a la composición de este órgano. Como he señalado en otro lugar ${ }^{36}$, este Tratado, en definitiva, se limitó a hacer hueco en las instituciones y órganos de la Unión a los países que se iban a incorporar, pero nada más.

Finalmente, el Tratado Constitucional tampoco ha modificado el ámbito competencial del Comité de las Regiones. Las materias de consulta obligatoria siguen siendo las que ya conocemos:

${ }^{36}$ «El Tratado de Niza...», cit., p. 2. 
- Empleo: orientaciones y medidas de fomento para propiciar la cooperación entre los Estados miembros y apoyar su acción en este ámbito (artículos III-206 y III-207).

- Política social: normas destinadas a alcanzar los objetivos en esta materia (artículo III-210).

- Fondo Social Europeo: medidas de aplicación (artículo III-219).

- Cohesión económica, social y territorial: medidas específicas (artículo III-221).

- Fondo Europeo de Desarrollo Regional: (artículos III-223 y III-224).

- Medio ambiente (artículo III-234).

- Transportes (artículo III-236).

- Navegación marítima y aérea (artículo III-245).

- Redes transeuropeas: orientaciones y restantes medidas (artículo III247).

- Energía: medidas para alcanzar los objetivos de esta política (artículo III-256).

— Salud pública (artículo III-278).

- Cultura (artículo III-280).

- Educación, juventud, deportes y formación profesional (artículos III282 y III-283).

Como se puede observar, tampoco en esta cuestión la Constitución para Europa supone un paso adelante, según venimos tratando de demostrar. El ámbito competencial que el nuevo Tratado atribuye a este órgano es el mismo que venía ejerciendo desde la reforma que experimentó como consecuencia del Tratado de Ámsterdam, que era $-\mathrm{y}$ así estaba ya previsto en el propio Tratado de la Unión Europea en su versión de Maastricht— una revisión de éste.

\section{Alguna pequeña incorporación}

Sólo alguna pequeña incorporación se aprecia en el nuevo Tratado que responde a una vieja aspiración del Comité de las Regiones. Como se recordará, el Comité siempre ha buscado que se le otorgara una legitimación activa privilegiada en los mismos términos reconocidos al Parlamento Europeo y al Banco Central Europeo en el caso de los recursos de nulidad y de inac- 
tividad. Pues bien, el artículo III-365.3 viene a satisfacer, si bien sólo de manera parcial, esa vieja pretensión.

El precepto citado no le reconoce - como hubiera sido su deseo- una condición de demandante privilegiado, esto es, la legitimación activa que sí tienen otras Instituciones de la Unión para impugnar los actos o la ausencia de éstos de las Instituciones dotadas de poder legisferente, aunque sí le iguala al Tribunal de Cuentas y al Banco Central Europeo en el marco del proceso de nulidad con el fin de salvaguardar sus prerrogativas.

Y, por otra parte, el artículo 8 del Protocolo $\mathrm{n}^{\circ}$ 2, anejo al Tratado, sobre la aplicación de los principios de subsidiariedad y proporcionalidad, señala que, de conformidad con los procedimientos establecidos en el artículo III365 , el CdR podrá interponer recursos contra actos legislativos europeos para cuya adopción la Constitución requiera su consulta. Se responde así, también de manera parcial y limitada, a su vocación de convertirse en el guardián del principio de subsidiariedad.

Se trata — como bien ha explicado MANGas MARTín ${ }^{37}$ - de dos preceptos ligados pero de alcance distinto. Mientras que el primero le da acceso limitado- al Tribunal en defensa de sus prerrogativas en el proceso decisional, en el segundo le faculta en relación con los actos en que fue consultado a controlar el modo de ejercicio de la competencia.

\section{UNA CONSIDERACIÓN FINAL}

Como sabemos, los ciudadanos franceses y holandeses — vía referéndumse han manifestado contrarios a la ratificación del Tratado por el que se establece una Constitución para Europa, y lo han hecho, además, de una manera contundente, pues se han pronunciado a favor del «no» el 55\%, con un $70 \%$ de participación en Francia y el 62\%, con un 68,2\% de participación en Holanda.

A partir de aquí, y ante la falta real de un plan «B» para el supuesto de que algunos Estados miembros negaran la ratificación del Tratado, las alarmas en Bruselas se han disparado y ha comenzado la búsqueda de salidas a la euroesclerosis que puede padecer esta organización.

Una de estas soluciones propugna introducir aquellos elementos del Tratado constitucional que, por consenso, pudieran ratificar los Estados miembros a través de los respectivos procedimientos nacionales o, incluso, que no precisaran - mejor aún- reforma alguna del Derecho originario. Es lo que se llama un «Tratado de Niza Plus» o, menos eufemísticamente, «meter la Constitución a pildorazos».

${ }^{37}$ La Constitución..., cit., p. 132. 
Pues bien, en esta improvisada respuesta a la situación actual no aparece por ningún lugar el Comité de las Regiones, y ello, a mi juicio, por dos razones.

La primera es que, como hemos venido tratando de demostrar, ningún cambio importante y significativo se produce en el nuevo Tratado, por lo que nada nuevo precisa introducirse. Las únicas incorporaciones que se contiene, como hemos visto, no son ni tan relevantes ni tan urgentes como para que necesite realizarse ninguna operación jurídica de cara a su aplicación lo más inmediata posible.

La segunda razón es más de fondo, pues, pese a la aparente sensibilización por el hecho regional y el reconocimiento de esta dimensión en la Unión Europea, lo cierto es que en la práctica no se va más allá que de oír a este órgano que, además, es lo suficientemente heterogéneo como para que pierda su coherencia y unidad en las consultas que emite, siendo muchas de ellas más un compromiso de las diferentes instancias territoriales que allí se dan cita ahí que de una verdadera dimensión regional.

En mi opinión, sólo una reforma auténtica y profunda de este órgano daría verosimilitud a la concienciación regional que se desprende del texto del nuevo Tratado. En suma, las bases ideológicas están sentadas, pero falta llevarlas a la práctica. 